\title{
A Simple, Enzymatic Biotransformation Method Using Fresh Green Tea Leaves Efficiently Generates Theaflavin-Containing Fermentation Water That Has Potent Physiological Functions in Mice and Humans
}

\author{
Masumi Takemoto, ${ }^{* a}$ Hiroaki Takemoto, ${ }^{b}$ Haruka Kouno, ${ }^{a}$ Tomomi Soeda, ${ }^{a}$ and \\ Takamichi Moriya $^{a}$ \\ ${ }^{a}$ School of Pharmaceutical Sciences, Ohu University; 31-1 Tomitamachi-Aza Misumido, Koriyama 963-8611, \\ Japan: and ${ }^{b}$ School of Pharmaceutical Sciences, Kitasato University; 5-9-1 Shirokane, Minato-ku, Tokyo 108-8641, \\ Japan. \\ Received January 19, 2017; accepted March 21, 2017; advance publication released online April 1, 2017
}

The polyphenolic compound theaflavin, the main red pigment in black tea, possesses many beneficial properties, such as fat-reducing and glucose-lowering capabilities. To produce theaflavin-containing fermentation water on a large scale, we have developed a simple, inexpensive, and selective enzymatic biotransformation method to obtain sufficient levels from fresh green tea leaves. Subsequent administration of theaflavin-containing fermentation water to obese mice on a high-fat diet inhibited body weight gain, decreased casual blood glucose and fasting blood glucose levels, and lowered mesenteric and total fat composition. To note, there were no significant differences observed in food consumption between the experimental and control (water without theaflavin) mice groups. Next, we investigated the effect of this water on blood glucose levels in healthy humans and found that it significantly inhibited blood glucose levels. Thus, we showed that theaflavin-containing fermentation water can be efficiently generated from fresh green tea leaves and demonstrated its significantly potent effects in vivo.

Key words theaflavin; fresh green tea leaf; peroxidase; hydrolase; polyphenol oxidase

Theaflavin (TF) (1) and its galloyl esters are the main red pigments in black tea. Their chemical structures [TF (1), TF 3-O-gallate (TF3G) (2), TF 3'-O-gallate (TF3'G) (3), and $\mathrm{TF}$ 3,3'-di-O-gallate (TFDG) (4)] are shown below (Fig. 1). These compounds are produced from their parent catechins [epicatechin (EC) (5), epicatechin 3-O-gallate (ECG) (6), epigallocatechin (EGC) (7), and epigallocatechin 3-O-gallate (EGCG) (8)] by polyphenol oxidase (PPO) or peroxidase (POD) in fresh green tea leaves during production of black tea leaves or green tea fermentation. ${ }^{1,2)}$

Previously, we had identified the enzymes involved in generation of TF (1) and its galloyl esters [TF3G (2), TF3'G (3), and TFDG (4)]. PPO was critical for production of TF galloyl esters [TF3G (2), TF3'G (3), and TFDG (4)], and POD was essential for TF (1) production. ${ }^{3)}$ Furthermore, via POD and hydrolase from Camellia sinensis cell culture, we succeeded in the selective, domino-type, one-pot enzymatic biotransformation method to convert four kinds of catechins [EC (5), ECG (6), EGC (7), and EGCG (8)] into TF (1) ${ }^{4)}$ (Fig. 2). C. sinensis cell culture is a rich source of $\mathrm{POD}^{5)}$ and a moderately rich source of hydrolase. In the one-pot reaction, EC (5) and
EGC (7) were immediately converted to TF (1) by endogenous POD. When the amount of EC (5) and EGC (7) in the reaction mixture sufficiently decreased, the $C$. sinensis cell culture hydrolase initiated hydrolysis of ECG (6) and EGCG (8) to produce EC (5), EGC (7), and gallic acid (9). Importantly, the hydrolysis reaction is an equilibrium reaction. It is thought that a hydrolysis reaction of EGCG (8) and ECG (6) progresses to make up for EC (5) and EGC (7) in the reaction mixture immediately after TF (1) generation from EC (5) and EGC (7). The EC (5) and EGC (7) generated by the hydrolysis reaction in turn is converted to TF (1) by POD. Thus, TF (1) synthesis and hydrolysis of ECG (6) and EGCG (8) are repeated to yield TF (1) and gallic acid (9) from EC (5), EGC (7), ECG (6), and EGCG (8) in the one-pot reaction. However, it was necessary to prepare the raw materials, because $C$. sinensis cell culture do not contain the four kinds of catechins [EC (5), EGC (7), ECG (6), and EGCG (8)].

To produce TF (1) using a simple method, we focused our efforts on fresh green tea leaves or frozen fresh green tea leaves containing the four major epi-type catechins [EC (5), ECG (6), EGC (7), and EGCG (8)] and enzymes (PPO, POD,<smiles>[R20]O[C@H]1Cc2c(O)cc(O)cc2O[C@H]1c1cc(O)c(O)c2c(=O)c(O)cc([C@H]3Oc4cc(O)cc(O)c4C[C@H]3O[R16])cc12</smiles>

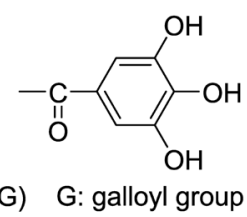

Fig. 1. Chemical Structures of TF (1), TF3G (2), TF3'G (3), and TFDG (4) 
and hydrolase). However, the enzymatic makeup in fresh or frozen green tea leaves varies greatly from that in $C$. sinensis cell culture. Particularly, $C$. sinensis cell culture has POD and hydrolase, and fresh or frozen fresh green tea leaves have PPO, POD and hydrolase. When POD and PPO exist, PPO predominates. There has yet to be a study indicating that the production of TF (1) can be selectively performed via use of frozen or fresh green tea leaves.

Toward this end, we investigated how to inactivate PPO while retaining POD activity. We developed a stationary method to activate POD and hydrolase at $25^{\circ} \mathrm{C}$. ${ }^{6}$ In this stationary method, TF (1) could be selectively and efficiently obtained without catechins from pulverized fresh or frozen fresh green tea leaves left to ferment in water for $120 \mathrm{~h}$ at $25^{\circ} \mathrm{C}$. Generally, the four major epi-type catechins [EC (5), ECG (6), EGC (7), and EGCG (8)] are enzymatically or non-enzymatically oxidized to produce quinones, which further provide various oxidized compounds. ${ }^{7,8)}$ Similarly, TF (1) are also obtained via

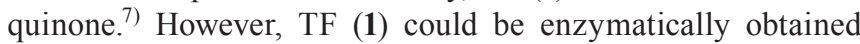
selectively and efficiently using this stationary method. Because, PPO, POD, and hydrolases from green tea leaves are released into the water when frozen fresh green tea leaves are pulverized in the presence of water.

However, this process was time consuming. Thus, we developed a semi-anaerobic stirring method. ${ }^{6}$ In this semianaerobic stirring method, TF (1) could be selectively obtained from pulverized frozen fresh green tea leaves, which were then stirred in water for $40 \mathrm{~min}$ at low speed $(300 \mathrm{rpm})$ to prevent contamination the air and yet achieve fermentation.

Using TF-containing fermentation water prepared by the semi-anaerobic stirring method, physiological effects of TFcontaining fermentation water were investigated in mice and humans. Particularly, the effects of the water on obesity were investigated in a high-fat diet-induced obesity mouse model for 40 weeks. Additionally, how TF-containing fermentation water affects human blood glucose levels were evaluated using the oral glucose tolerance test. These results and their implications are discussed.

\section{MATERIALS AND METHODS}

HPLC Analysis HPLC analysis of TF (1), TF3G (2), TF3'G (3), TFDG (4), EC (5), ECG (6), EGC (7), and EGCG (8) was performed with a JASCO HPLC system (PU-980, UV-970) and a $4.6 \times 250 \mathrm{~mm}$ ODS120A column (TOSOH) under the following conditions: solvent, acetonitrile-ethyl acetate (AcOEt) $-0.05 \% \mathrm{H}_{3} \mathrm{PO}_{4}$ at $21: 3: 76$; flow rate, $1.0 \mathrm{~mL} / \mathrm{min}$; temperature, $25^{\circ} \mathrm{C}$ and $\mathrm{UV}$ detection at $280 \mathrm{~nm}$.

Materials We collected fresh green tea leaves in June from Shinma, Shizuoka City, Shizuoka Prefecture, Japan. These leaves were preserved in a freezer at $-20^{\circ} \mathrm{C}$. HighFat Diet 32 (HFD32) was purchased from CLEA Japan, Inc., Japan. Commercial green tea beverage (Honsabou) was purchased from Otsuka Beverage Co., Ltd., Japan. Commercial black tea beverage (JAVA tea) was purchased from Otsuka Beverage Co., Ltd., Japan. Glucose was purchased from Yoshida Pharmaceutical Co., Ltd., Japan. Blood glucose levels were measured with Medisafe mini (Terumo Co., Inc., Japan).

Stationary Method to Obtain TF-Containing Fermentation Water Using Frozen Fresh Green Tea Leaves and Water Fresh green tea leaves, preserved by freezing at $-20^{\circ} \mathrm{C}(10 \mathrm{~g})$, were added to water $(100$ or $800 \mathrm{~mL})$ and pulverized with an industrial mixer for 1 or $3 \mathrm{~min}$ at $25^{\circ} \mathrm{C}$. After pulverization, the mixture was allowed to stand for $24 \mathrm{~h}$ or $120 \mathrm{~h}$ at $25^{\circ} \mathrm{C}$ and then was filtered. Quantification of filtrate [ECG (6), EGCG (8), TF (1), TF3G (2), TF3'G (3), TFDG (4), and gallic acid (9)] was performed by the calibration curve of the corresponding authentic compounds [ECG (6), EGCG (8), TF (1), TF3G (2), TF3'G (3), TFDG (4), and gallic acid (9)].

Semi-naerobic Stirring Method to Obtain TF-Containing Fermentation Water Using Frozen Fresh Green Tea Leaves and Water Fresh green tea leaves, preserved by freezing at $-20^{\circ} \mathrm{C}(120 \mathrm{~g})$, were added to water $(4 \mathrm{~L})$ and pulverized with an industrial mixer for $1 \mathrm{~min}$ at $25^{\circ} \mathrm{C}$. After pulverization, the mixture was moved to a $30 \mathrm{~L}$ stainless steel tank. We repeated these operation four times. After all frozen fresh green tea leaves $(480 \mathrm{~g})$ were pulverized, water $(9 \mathrm{~L})$ was added to the mixture. Afterwards, the mixture was stirred with an industrial stirrer at low speed $(300 \mathrm{rpm})$ for $40 \mathrm{~min}$ at $25^{\circ} \mathrm{C}$. The mixture was filtrated and retort sterilized.

Composition Analysis of the TF-Containing Fermentation Water from the Semi-anaerobic Stirring Method Composition analysis of the TF-containing fermentation water was performed by Ecopro-research Co., Ltd. Japan. Component values $(\mathrm{mg} / 100 \mathrm{~mL})$ were as follows: aspartic acid (0.01), glutamic acid (0.01), asparagine (0), serine (0.02), glutamine $(0.05)$, threonine $(0.09)$, arginine $(0.01)$, theanine $(0.63)$, alanine (0.06), tyrosine $(0.02)$, methionine $(0)$, valine $(0)$, phenylalanine $(0.01)$, isoleucine $(0.01)$, leucine $(0.01)$, lysine (0), $\gamma$-aminobutyric acid (GABA) (0.17), caffeine (10.7), gallic acid (6.3), polyphenol (89.2), TF (2.0) and saponin (26.2). The total polyphenol content was analyzed with Folin-Denis assay using tannic acid as a standard. Saponin content was analyzed with the phenol-sulfuric acid method using a commercially available saponin in tea seed as a standard.

Composition Analysis of Commercial Black Tea Beverage Composition analysis of commercial black tea beverage was performed by Ecopro-research Co., Ltd. Component values $(\mathrm{mg} / 100 \mathrm{~mL})$ were as follows: aspartic acid $(0.65)$, glutamic acid (0.82), asparagine (0.24), serine (0.34), glutamine (0.37), threonine (0.07), arginine (0.09), theanine (3.5), alanine (1.09), tyrosine $(0.24)$, methionine $(0.16)$, valine $(0.06)$, phenylalanine $(0.23)$, isoleucine $(0.29)$, leucine $(0.1)$, lysine $(0.09)$, GABA (0.1), GC (0), EGC (1.5), C (0.3), EC (0.2), EGCG (2.0), GCG (0), ECG (0.3), CG (0), caffeine (14.6), gallic acid (3.6), polyphenol (62.4), TF (0), and saponin (29.5). The total polyphenol content was analyzed with Folin-Denis assay using tannic acid as a standard. Saponin content was analyzed with the phenol-sulfuric acid method using a commercially available saponin in tea seed as a standard.

Composition Analysis of Commercial Green Tea Beverage Composition analysis of commercial green tea beverage was performed by Ecopro-research Co., Ltd. Component values $(\mathrm{mg} / 100 \mathrm{~mL})$ were as follows: aspartic acid (1.14), glutamic acid (1.53), asparagine (0.03), serine (0.38), glutamine $(0.22)$, threonine (0.59), arginine (0.06), theanine (5.3), alanine (1.07), tyrosine $(0.2)$, methionine $(0.2)$, valine $(0.06)$, phenylalanine $(0.12)$, isoleucine $(0.14)$, leucine $(0.1)$, lysine $(0.08)$, GABA (0.1), GC (0), EGC (7.1), C (2.7), EC (2.2), EGCG (7.9), GCG (0), ECG (2), CG (0), caffeine (12.6), gallic acid (0.8), polyphenol (77.9), TF (0), and saponin (26.4). The total polyphenol content was analyzed with Folin-Denis assay using tannic 
acid as a standard. Saponin content was analyzed with the phenol-sulfuric acid method using a commercially available saponin in tea seed as a standard.

Animals Four-week-old male C57BL6 mice were purchased from Japan SLC, Inc. (Japan). Prior to the experiments, the mice were acclimatized for 1 week in a $23 \pm 1^{\circ} \mathrm{C}$ environment with $55 \pm 5 \%$ humidity and a $12 \mathrm{~h}$ light-dark cycle. The study was conducted in accordance with the guidelines for animal care, handling and termination from the University of Shizuoka, which are aligned with both international and Japanese guidelines on animal care and welfare.

Feeding After 1 week of acclimatization, healthy mice were separated into a control water group $(n=10)$, TF-containing fermentation water group $(n=10)$, commercial green tea beverage group $(n=10)$, and commercial black tea beverage group $(n=10)$. All groups were provided with a high-fat diet (High Fat Diet 32) ad libitum and either control water, TFcontaining fermentation water, commercial green tea beverage, or commercial black tea beverage for 40 weeks.

Oral Glucose Tolerance Test in Healthy Human Individuals Research involving human individuals was approved by the Ohu University Research Ethics Committee based on the Declaration of Helsinki. In this study, we obtained informed content. Eight healthy adults (three men and five women) were enrolled. Their age range was from 23 years old to 59 years old. We used water as the control, TF-containing fermentation water as the experimental, and none-TF water as a placebo. The wash out of either water was for 1 week, and we performed a crossover examination. Human individuals fasted from 10 p.m. the previous day, and fasting blood glucose levels were measured at 10 a.m. After all individuals consumed $300 \mathrm{~mL}$ of either water, TF-containing fermentation water or placebo, in which was dissolved $50 \mathrm{~g}$ of glucose, had their blood glucose levels measured at 15, 30, 45, 60, 75, 90, and $120 \mathrm{~min}$ after administration. Next, six patients consumed $300 \mathrm{~mL}$ of either water, TF-containing fermentation water or placebo, for 1 week, 3 times a day. On the day following final consumption of their beverages, glucose $(50 \mathrm{~g}$ dissolved in $300 \mathrm{~mL}$ of water) was administered, and blood glucose levels were measured at $15,30,45,60,75,90$, and $120 \mathrm{~min}$ after administration. Next, $300 \mathrm{~mL}$ of TF-containing fermentation water were consumed by five human individuals for 4 weeks, 3 times a day. On the day following final consumption of the TF-containing water, glucose $(50 \mathrm{~g}$ dissolved in $300 \mathrm{~mL}$ of water) was administered, and blood glucose levels were measured at $15,30,45,60,75,90$, and $120 \mathrm{~min}$ after administration. Next, $300 \mathrm{~mL}$ of water were consumed by five human individuals for 4 weeks, 3 times a day. On the day following final consumption of the water, glucose $(50 \mathrm{~g}$ dissolved in $300 \mathrm{~mL}$ of water) was administered, and blood glucose levels were measured at $15,30,45,60,75,90$, and $120 \mathrm{~min}$ after administration.

Manufacturing Method of Placebo, Non-TF Water for Human Studies In this study, $200 \mathrm{~g}$ of frozen, fresh green tea leaves were dipped into boiling water to inactivate endogenous enzymes. We added $3 \mathrm{~L}$ water to the boiled leaves and pulverized them with an industrial mixer for $1 \mathrm{~min}$ at $25^{\circ} \mathrm{C}$. We added water until the final volume was $5 \mathrm{~L}$. The mixture was stirred with an industrial stirrer at low speed (300 rpm) for $40 \mathrm{~min}$ at $25^{\circ} \mathrm{C}$. The mixture was filtered and retort sterilized.
Composition Analysis of Placebo, Non-TF Water for Human Studies Composition analysis of non-TF water as a placebo was produced by Ecopro-research Co., Ltd. Component values $(\mathrm{mg} / 100 \mathrm{~mL})$ were as follows: EGCG (28.6), ECG (5.3), EC (5.3), EGC (21.8), aspartic acid (1.5), glutamic acid (3.0), asparagine (0.43), serine (0.2), glutamine (0.9), threonine (0.9), arginine (n.d.), theanine (7.8), alanine (1.7), tyrosine $(0.4)$, methionine $(0)$, valine $(0.1)$, phenylalanine $(0)$, isoleucine (0.8), leucine (0.08), lysine (0), GABA (1.8), caffeine (20.5), gallic acid (1.4), polyphenol (68.6), and saponin (26.2). The total polyphenol content was analyzed with Folin-Denis assay using tannic acid as a standard. Saponin content was analyzed with the phenol-sulfuric acid method using a commercially available saponin in tea seed as a standard.

Manufacturing Method of TF-Containing Fermentation Water for Human Studies We added $3 \mathrm{~L}$ water to $200 \mathrm{~g}$ of frozen green tea leaves and pulverized them with an industrial mixer for $1 \mathrm{~min}$ at $25^{\circ} \mathrm{C}$. We added water until the final volume was $5 \mathrm{~L}$. The mixture was stirred with an industrial stirrer at low speed $(300 \mathrm{rpm})$ at $25^{\circ} \mathrm{C}$ to prevent contamination of air for $40 \mathrm{~min}$. The mixture was filtrated and retort sterilized.

Composition Analysis of TF-Containing Fermentation Water for Human Studies Composition analysis was the TF-containing fermentation water was performed by Ecoproresearch Co., Ltd. Component values $(\mathrm{mg} / 100 \mathrm{~mL})$ were as follows: aspartic acid (0.02), glutamic acid (0.02), asparagine $(0)$, serine $(0.04)$, glutamine $(0.1)$, threonine $(0.18)$, arginine (0.02), theanine (1.31), alanine (0.12), tyrosine (0.04), methionine $(0)$, valine $(0)$, phenylalanine $(0.02)$, isoleucine $(0.02)$, leucine (0.02), lysine (0), GABA (0.35), caffeine (22.3), gallic acid (13.1), polyphenol (185.8), TF (4), and saponin (52.0). The total polyphenol content was analyzed with Folin-Denis assay using tannic acid as a standard. Saponin content was analyzed with the phenol-sulfuric acid method using a commercially available saponin in tea seed as a standard.

Statistical Analyses All data are presented as mean \pm standard deviation (S.D.). Group differences were statistically analyzed for significances as follows: Bonferroni's parametric multiple comparison test was used after Bartlett's analysis for experiments comparing one control group with two or three experimental groups. $p<0.05$ was considered statistically significant.

\section{RESULTS}

Stationary Method to Obtain TF (1) and TF-Containing Fermentation Water We investigated the ratio of water volumes to the weights of frozen fresh green tea leaves, pulverizing time, and stationary time.

Product [TF (1), TF3G (2), TF3'G (3), and TFDG (4)] and residual starting materials [EGCG (8) and EC (5)] are listed in Table 1 . When we used 10 volumes of water to weight of frozen fresh green tea leaves, pulverized with an industrial mixer for $1 \mathrm{~min}$ at $25^{\circ} \mathrm{C}$, and allowed the mixture to stand for $120 \mathrm{~h}$ at $25^{\circ} \mathrm{C}$, the residual starting materials were absent, and TF (1) (44 mg) was obtained as sole product (entry 2). When we used 80 volumes of water to weights of frozen fresh green tea leaves, pulverized for $1 \mathrm{~min}$ at $25^{\circ} \mathrm{C}$, and allowed mixture to stand for $120 \mathrm{~h}$ at $25^{\circ} \mathrm{C}$, TF (1) $(85 \mathrm{mg})$ was also obtained as the sole product (entry 3 ). When frozen fresh green tea leaves were pulverized for $3 \mathrm{~min}$, TF (1) was not selectively obtained 
Table 1. Stationary Method to Obtain TF (1) from Frozen Fresh Green Tea Leaves

\begin{tabular}{|c|c|c|c|c|c|c|c|c|c|c|}
\hline & $\begin{array}{l}\text { Leaves } \\
\text { (g) }\end{array}$ & $\begin{array}{l}\text { Water } \\
(\mathrm{mL})\end{array}$ & $\begin{array}{l}\text { Pulverizing time } \\
\text { (min) }\end{array}$ & $\begin{array}{l}\text { Stationary time } \\
\text { (h) }\end{array}$ & $\begin{array}{c}\mathrm{TF} \\
(\mathrm{mg})\end{array}$ & $\begin{array}{l}\text { TF3G } \\
(\mathrm{mg})\end{array}$ & $\begin{array}{l}\mathrm{TF} 3^{\prime} \mathrm{G} \\
(\mathrm{mg})\end{array}$ & $\begin{array}{l}\text { TFDG } \\
(\mathrm{mg})\end{array}$ & $\begin{array}{l}\text { EGCG } \\
(\mathrm{mg})\end{array}$ & $\begin{array}{l}\text { EGC } \\
(\mathrm{mg})\end{array}$ \\
\hline 1 & 10 & 100 & 1 & 24 & 7.5 & 1.4 & 0.8 & 0.39 & 390 & 8.1 \\
\hline 2 & 10 & 100 & 1 & 120 & 44 & 0 & 0 & 0 & 0 & 0 \\
\hline 3 & 10 & 800 & 1 & 120 & 85 & 0 & 0 & 0 & 0 & 0 \\
\hline 4 & 10 & 100 & 3 & 24 & 29 & 7.0 & 4.2 & 3.4 & 310 & 4.0 \\
\hline 5 & 10 & 100 & 3 & 120 & 40 & 2.9 & 1.5 & 0.9 & 0 & 0 \\
\hline 6 & 10 & 800 & 3 & 120 & 70 & 9 & 2.5 & 3.8 & 0 & 0 \\
\hline
\end{tabular}

Table 2. Urine and Fecal Amounts in 18-Week-Old Mice

\begin{tabular}{lllr}
\hline \hline & Control & Black tea & Green tea \\
\hline Feces $(\mathrm{g})$ & $0.22 \pm 0.03$ & $0.23 \pm 0.03$ & $0.20 \pm 0.02$ \\
Urine $(\mathrm{g})$ & $0.49 \pm 0.06$ & $0.49 \pm 0.09$ & $0.50 \pm 0.03$ \\
\hline
\end{tabular}

All data are presented as the mean \pm S.D. of each group $(n=10)$. Multiple-comparison tests with the Bonferroni correction were performed. $* * p<0.05$. TF-water: TF-containing fermentation water.

Table 3. Rate of Body Weight Gain (\%)

\begin{tabular}{rrrr}
\hline \hline & Control & Black tea & Green tea \\
\hline 8-Weeks of age & $81.0 \pm 3.4$ & $91.9 \pm 4.6$ & $82.8 \pm 4.2$ \\
12-Weeks of age & $141 \pm 3.9$ & $144 \pm 3.1$ & $139 \pm 2.3$ \\
16-Weeks of age & $174 \pm 3.6$ & $171 \pm 5.5$ & $164 \pm 2.6$ \\
24-Weeks of age & $189 \pm 4.8$ & $189 \pm 4.3$ & $136 \pm 2.3$ \\
36-Weeks of age & $215 \pm 5.5$ & $214 \pm 4.5$ & $174 \pm 4.4^{*}$ \\
\hline
\end{tabular}

All data are presented as mean \pm S.D. of each group $(n=10)$. Multiple-comparison tests with the Bonferroni correction were performed. $* p<0.05$. TF-water: TF-containing fermentation water.

Table 4. Casual Blood Glucose Levels (mg/dL)

\begin{tabular}{llll}
\hline \hline Age & Control & Black tea & Green tea \\
\hline 12-Weeks & $196.1 \pm 13.6$ & $183.1 \pm 11.9$ & $203.2 \pm 13.6$ \\
16-Weeks & $217.7 \pm 12.5$ & $195.8 \pm 13.7$ & $212.4 \pm 16.3$ \\
24-Weeks & $190.7 \pm 9.2$ & $199.2 \pm 9.3$ & $203.5 \pm 6.5$ \\
36-Weeks & $192.0 \pm 9.5$ & $175.2 \pm 7.7$ & $177.9 \pm 5.9$ \\
\hline
\end{tabular}

All data are presented as the mean \pm S.D. of each group $(n=10)$. Multiple-comparison tests with the Bonferroni correction were performed. TF-water: TF-containing fermentation water.

(entry 4-6).

Semi-anaerobic Stirring Method to Obtain TF (1) and TF-Containing Fermentation Water As outlined in the methods above, this method was faster. Via HPLC analysis, TF (1) (1.7g), gallic acid (2.4g) and caffeine (3.6g) were yielded per $480 \mathrm{~g}$ of green tea leaves, which were preserved by freezing. Polyphenolic quantity was $6.1 \mathrm{~g}$.

Effect of TF-Containing Fermentation Water on Fat Accumulation in a High-Fat Diet-Induced Obesity Mouse Model A high-fat diet-induced obesity mouse model was used to investigate the effects of TF-containing fermentation water. In this study, 4-week-old healthy C57BL6 male mice were acclimatized for 1 week before being randomly assigned into the experimental groups. The groups were provided with either control water, TF-containing fermentation water, commercial black tea beverage, or commercial green tea beverage. All groups were fed a high-fat diet (HFD 32) ad libitum for 40 weeks. There was no significant difference in food consumption ( $3.4 \mathrm{~g}$ per day) among the groups, and liquid consumption was also approximately equal ( $2 \mathrm{~mL}$ per day). Furthermore, no significance difference in urine production was observed (Table 2). However, fecal amounts in the TF-containing fermentation water mouse group was significantly higher.

We estimated rate of body weight gain based on the body weight at 5 weeks of age (Table 3). TF-containing fermentation water significantly decreased body weight gain after 16 weeks of age.

Casual blood glucose levels were measured at 1 p.m. at 12, 16, 24 and 36 weeks of age (Table 4). At 12, 16, and 24 weeks of age, TF-containing fermentation water tended to reduce blood glucose levels in mice.

When the mice were 36 - or 40 -weeks of age, $14 \mathrm{~h}$ fasting blood glucose levels were measured (Table 5). TF-containing fermentation water significantly decreased fasting blood glucose levels in 36 or 40 week old mice.

After $14 \mathrm{~h}$ of fasting at 40 -weeks of age, each mouse was anesthetized with diethyl ether, and the abdomen was sectioned. The perirenal, epididymal, and mesenteric fat were 
Table 5. Fasting Blood Glucose Levels $(\mathrm{mg} / \mathrm{dL})$

\begin{tabular}{lcccc}
\hline \hline Age & Control & Black tea & Green tea & TF-water \\
\hline 36 -Weeks & $181.9 \pm 5.8$ & $161.7 \pm 2.2^{*}$ & $171.7 \pm 4.0$ & $155.6 \pm 3.3^{*}$ \\
40 -Weeks & $165.2 \pm 5.9$ & $158.8 \pm 8.4$ & $156.3 \pm 7.3$ & $136.0 \pm 5.0^{*}$ \\
\hline
\end{tabular}

All data are presented as mean \pm S.D. of each group $(n=10)$. Multiple-comparison tests with the Bonferroni correction were performed. $* p<0.05$. TF-water: TF-containing fermentation water.

Table 6. Fat Compositions (g)

\begin{tabular}{|c|c|c|c|c|}
\hline & Control & Black tea & Green tea & TF-water \\
\hline Perirenal fat & $2.89 \pm 0.23$ & $2.75 \pm 0.19$ & $2.64 \pm 0.23$ & $2.65 \pm 0.11$ \\
\hline Epididymal fat & $1.24 \pm 0.087$ & $1.03 \pm 0.05$ & $1.36 \pm 0.14$ & $1.08 \pm 0.14$ \\
\hline Mesenteric fat & $1.18 \pm 0.069$ & $1.02 \pm 0.094$ & $1.12 \pm 0.07$ & $0.85 \pm 0.048 *$ \\
\hline Total fat & $5.30 \pm 0.35$ & $4.80 \pm 0.26$ & $5.06 \pm 0.36$ & $4.58 \pm 0.11 *$ \\
\hline
\end{tabular}

All data are presented as the mean \pm S.D. of each group $(n=10)$. Multiple-comparison tests with the Bonferroni correction were performed. $* p<0.05$. TF-water: TF-containing fermentation water.

Table 7. Oral Glucose Tolerance Tests, Single Water Doses $(n=8)$

\begin{tabular}{lcc}
\hline \hline & Control & TF-water \\
\hline Maximum blood glucose level $(\mathrm{mg} / \mathrm{dL})$ & $172 \pm 5.3$ & $153 \pm 18.3^{*}$ \\
$\Delta A U C_{\mathrm{g}, 0-120 \mathrm{~min}}^{*}$ & 100 & $70.1 \pm 25.7 *$ \\
\hline
\end{tabular}

All data are presented as the mean \pm S.D. of each group $(n=10)$. Multiple-comparison tests with the Bonferroni correction were performed. $* p<0.05$. TF-water: TF-containing fermentation water.

Table 8. Oral Glucose Tolerance Tests $(n=6)$ after 1 Week

\begin{tabular}{lcr}
\hline \hline & Control & TF-water \\
\hline Maximum blood glucose level (mg/dL) & $163 \pm 19.1$ & $159 \pm 28.5$ \\
$\Delta A U C_{\mathrm{g}, 0-120 \mathrm{~min}}^{*}$ & 100 & $80.4 \pm 14.0$ \\
\hline
\end{tabular}

All data are presented as the mean \pm S.D. of each group $(n=10)$. Multiple-comparison tests with the Bonferroni correction were performed. TF-water: TF-containing fermentation water.

Table 9. Oral Glucose Tolerance Tests $(n=5)$ after Consumption of TF-Containing Fermentation Water for 1 Month and the Following Consumption of Water for 1 Month

\begin{tabular}{lcr}
\hline \hline & Start time & TF-water for 1 month \\
\hline Maximum blood glucose level $(\mathrm{mg} / \mathrm{dL})$ & $187 \pm 7.5$ & $161 \pm 7.2 * *$ \\
$\Delta A U C_{\mathrm{g}, 0-120 \mathrm{~min}}^{*}$ & 100 & $88.7 \pm 18.3$ \\
\hline
\end{tabular}

All data are presented as the mean \pm S.D. of each group $(n=10)$. Multiple-comparison tests with the Bonferroni correction were performed. $* p<0.05$; $* * p<0.05$. TF-water: TF-containing fermentation water.

immediately excised and weighed (Table 6). TF-containing fermentation water significantly decreased mesenteric and total fat in comparison to all other groups.

Effect of TF-Containing Fermentation Water on the Oral Glucose Tolerance Test in Healthy Human Individuals Oral glucose tolerance test $(n=8)$ results using the three different water types are presented in Table 7. $\Delta$ area under curve $(A U C)_{\mathrm{g}, 0-120 \mathrm{~min}}$ is expressed as relative value. $\triangle A U C_{\mathrm{g}, 0-120 \mathrm{~min}}$ $=A U C_{\mathrm{g}, 0-120 \mathrm{~min}}$ (orally glucose load teat) $-A U C_{\mathrm{g}, 0-120 \mathrm{~min}}$ (fasting blood glucose level). $\triangle A U C_{\mathrm{g}, 0-120 \mathrm{~min}}^{*}=\triangle A U C_{\mathrm{g}, 0-120 \mathrm{~min}}$ (TF-containg fermentation water or placebo) $/ \triangle A U C_{\mathrm{g}, 0-120 \mathrm{~min}}$ (control) $\times 100$. TF-containing fermentation water significantly reduced maximum blood glucose level and $\triangle A U C_{\mathrm{g}, 0-120 \mathrm{~min}}^{*}$ compared to control and placebo groups.

Oral glucose tolerance tests $(n=6)$ after consumption of each water type for one week are shown in Table 8. After one week of consumption (control, TF-containing fermentation water, placebo), TF-containing fermentation water tended to reduce maximum blood glucose levels and $\triangle A U C_{\mathrm{g}, 0-120 \min }^{*}$.

We show the results of maximum blood glucose level $(\mathrm{mg} / \mathrm{dL})$ and $\triangle A U C_{\mathrm{g}, 0-120 \mathrm{~min}}^{*}$ in Table 9 by the oral glucose tolerance tests after consumption of TF-containing fermentation water for 1 month and the following consumption of water for 1 month.

The inhibition of increase in maximum blood glucose values of TF-containing fermentation water was significantly observed by the glucose load test after consumption of the TF-containing fermentation water for 1 month, because the inhibition of increase in blood glucose values was not observed by the glucose load test after consumption of control (water) 


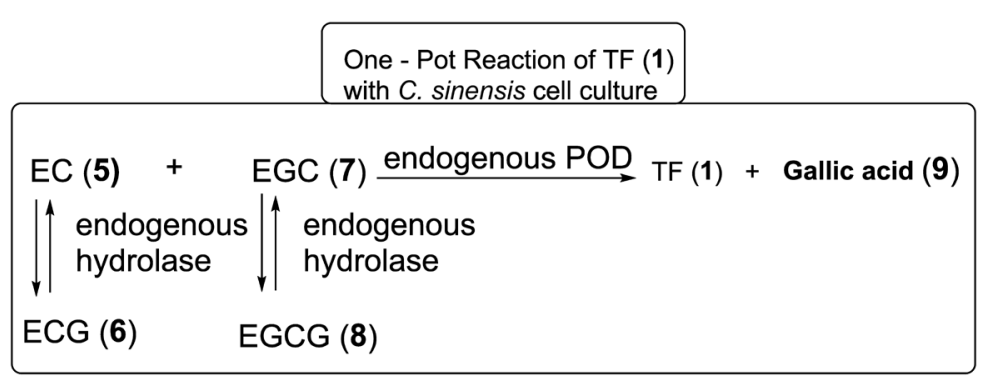

Fig. 2. One-Pot, Domino-Type Enzymatic Selective Synthesis of TF (1)

for 1 month.

\section{DISCUSSION}

In our previous studies, we found that POD was key for production of TF (1) while PPO was needed for production of TF (1), TF3G (2), TF3'G (3), and TFDG (4). However, there are no black tea leaves containing TF (1) alone, despite the presence of POD in fresh green tea leaves. It is thought that TF (1), TF3G (2), TF3'G (3), and TFDG (4) are produced when fresh green tea leaves are fermented during the black tea production process, because PPO acts primarily as compared to POD present in fresh green tea leaves.

In the present study, we developed an easy method to control PPO and POD in frozen, fresh green tea leaves. PPO catalyzes oxidation of substrates in the presence of oxygen. On the other hand, POD catalyzes oxidation of substrates in the presence of hydrogen peroxide. We focused on these differences. Based on our previous studies, PPO predominated compared to POD in fresh green tea leaves.

Oxygen blocking methods can inactivate PPO. The use of an argon atmosphere is a common method, but this method is expensive and difficult to perform. To create a more costeffective method, we pulverized fresh, frozen green tea leaves in the presence of a large quantity of water with a mixer for $1 \mathrm{~min}$ at $25^{\circ} \mathrm{C}$ and then allowed the mixture to stand at $25^{\circ} \mathrm{C}$. In the stationary method, PPO activity decreased as dissolved oxygen in water decreased. Meanwhile, POD activity was maintained. Thus, it was assumed that the reaction occurred in a POD-selective manner when the stationary method was used.

Because mixer pulverization incorporates air and increases the oxygen content, the PPO-mediated oxidation is also activated as the duration of pulverization is prolonged (Table 1). To note, water used in the present study was not degassed. Water was added to frozen fresh green tea leaves, and the leaves were pulverized with a mixer. Because the air was incorporated by this mixer pulverization, it was required to adjust the amount of water depending on whether the intended condition was to be PPO or POD-selective (Table 1). As for the reaction in a large quantity of water, if dissolved oxygen disappeared, then anaerobic conditions prevailed, rendering PPO inactive. Based on these results, TF (1) could be selectively and efficiently obtained without residual raw materials (catechins) by pulverizing frozen fresh green tea leaves with a mixer for $1 \mathrm{~min}$ at $25^{\circ} \mathrm{C}$ in the presence of 80 volumes of water, allowing mixture to stand for $120 \mathrm{~h}$ at $25^{\circ} \mathrm{C}$ (Table 1 , entry 3).

When frozen fresh green tea leaves are pulverized in the

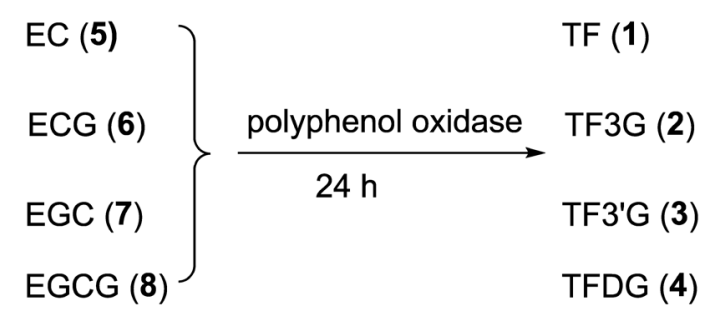

Fig. 3. Synthesis of TF (1), TF3G (2), TF3'G (3), and TFDG (4) with PPO

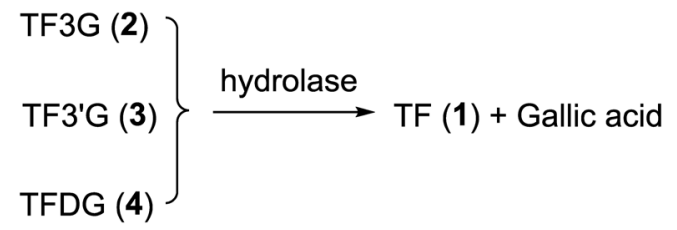

Fig. 4. Removal of the Gallate Group by Hydrolase

presence of water, PPO, POD, and hydrolases from green tea leaves as well as various components of green tea leaves, such as catechins and caffeine, are released into the water. Regardless of the amount of water and duration of the pulverizing, TF (1), TF3G (2), TF3'G (3), and TFDG (4) were yielded in the stationary method for $24 \mathrm{~h}$ at $25^{\circ} \mathrm{C}$ (Table 1, Entries 1, 4). We proposed that TF (1), TF3G (2), TF3'G (3), and TFDG (4) were yielded for $24 \mathrm{~h}$ at $25^{\circ} \mathrm{C}$ as shown in Fig. 3. PPO is activated in the water during the pulverization process for $1 \mathrm{~min}$.

When the pulverized material was allowed to stand for $120 \mathrm{~h}$ at $25^{\circ} \mathrm{C}$, PPO activity decreased as dissolved oxygen in the water was consumed. The resulting TF3G (2), TF3'G (3), and TFDG (4) underwent the removal of the gallate group by hydrolases to yield TF (1) and gallic acid (Fig. 4).

During pulverization, PPO, POD, and hydrogen peroxide were released into the water due to stress. While PPO no longer functioned once dissolved oxygen was consumed, the reaction was presumably driven by POD and hydrolase as shown in Fig. 2. In our previous report, ${ }^{4)} C$. sinensis cell culture have POD activity, and four catechins entirely and selectively converted into TF (1) by POD and hydrolases (Fig. 2). It is assumed that the reaction in the current study proceeded via a similar mechanism. When the pulverized material was allowed to stand for $120 \mathrm{~h}$, three reactions (Figs. 2-4) proceeded to selectively convert four catechin (5-8) into TF (1).

We investigated methods to shorten the reaction time to only $40 \mathrm{~min}$, and we showed that TF (1) could be successfully obtained using approximately $480 \mathrm{~g}$ of frozen fresh green tea leaves pulverized for $1 \mathrm{~min}$ in the presence of 50 volumes of water, with stirring at low speeds minimize introduction of 
air. In our semi-anaerobic stirring method, the one-pot domino-type enzymatic selective reaction progressed to obtain TF (1) as shown in Fig. 2. The advantages of this method is that it is simple and inexpensive, and it provides the means to largescale production of TF (1), which was vital for our physiological studies in mice and humans.

In this paper, physiological function in mice and humans were investigated with TF-containing fermentation water prepared with the semi-anaerobic stirring method. This is the first study showing the long-term anti-obesity effect (40 weeks) of TF-containing fermentation water in mice given a high fat diet. TF (1) $(0.04 \mathrm{mg} / \mathrm{d})$ for 40 weeks significantly inhibited body weight gain, casual and fasting blood glucose levels, and mesenteric and total fat composition. It is known that plant polyphenol inhibits enzymatic activities. ${ }^{9}$ (Additionally, a previous study ${ }^{10)}$ suggested that catechins inhibit amylase activity to control blood sugar levels in healthy human. TF [TF (1), TF3G (2), TF3'G (3), and TFDG (4)] had strong amylase inhibitory activity in vitro. ${ }^{11)}$ Regarding sucrase inhibitory activity, ECG (6), EGCG (8), TF (1), TF3G (2), TF3'G (3), and TFDG (4) possess inhibitory activity in vitro. ${ }^{12)}$ Our in vivo work presented here demonstrated that TF-containing fermentation water significantly reduced casual and fasting blood glucose levels, which agrees with past in vitro test. ${ }^{11,12)}$ We found that TF-containing fermentation water exhibited anti-obesity activities in mice. In healthy humans, TF-containing fermentation water suppressed hyperglycemia in a single dose and on consumption for 1 and 4 weeks.

In summary, we posit that fermented tea contains clinically relevant amounts of TF (1), making it a viable dietary supplement. Gallic acid, polyphenol, saponin and TF (1) are more included in TF-containing fermentation water compared with placebo. Triterpenoid saponin was reported to inhibit blood glucose levels, ${ }^{13)}$ and gallic acid decreased body weight and protected against hepatic steatosis in high-fat diet-induced nonalcoholic fatty liver disease mice. ${ }^{14)}$ Almost catechins [EC (5), EGC (7), ECG (6), and EGCG (8)] were produced to TF (1) in TF-containg fermentation water. Furthermore, it was possible that TF (1) was oxidized, but we were not able to confirm these oxidized compounds. We believe that the functionality of TF-containing fermentation water is caused not only by TF (1) but also by the synergistic effect with gallic acid and saponin. ${ }^{15)}$ Further studies are warranted to elucidate the underlying mechanisms of TF (1).

Acknowledgments This study was supported in part by a project of the Shizuoka Prefecture and Shizuoka City Collaboration of Regional Entities for the Advancement of Technological Excellence, Japan Science and Technology Agency (JST).

Conflict of Interest The authors declare no conflict of interest.

\section{REFERENCES}

1) Tanaka T, Mine C, Inoue K, Matsuda M, Kouno I. Synthesis of theaflavin from epicatechin and epigallocatechin by plant homogenates and role of epicatechin quinone in the synthesis and degradation of theaflavin. J. Agric. Food Chem., 50, 2142-2148 (2002).

2) Sang S, Yang CS, Ho C-T. Peroxidase-mediated oxidation of catechins. Phytochem. Rev., 3, 229-241 (2004).

3) Takemoto M, Takemoto H, Sakurada A. Synthesis of theaflavin with Camellia sinensis cell culture and inhibition of increase in blood sugar values in high-fat diet mice subjected to sucrose or glucose loading. Tetrahedron Lett., 55, 5038-5040 (2014).

4) Takemoto M, Takemoto H, Saijo R. Theaflavin synthesized in a selective, domino-type, one-pot enzymatic biotransformation method with Camellia sinensis cell culture inhibits weight gain and fat accumulation to high-fat diet-induced obese mice. Biol. Pharm. Bull., 39, 1347-1352 (2016).

5) Takemoto M, Aoshima Y, Stoynov N, Kutney JP. Establishment of Camellia sinensis cell culture with high peroxidase activity and oxidative coupling reaction of dibenzylbutanolides. Tetrahedron Lett., 43, 6915-6917 (2002).

6) Takemoto M. Method of producing fermented tea drink rich in theaflavins. UK Patent GB2471250, 2013.1.30. Japanese Patent 5472092, 2014.2.14

7) Tanaka T, Kouno I. Oxidation of tea catechins: chemical structures and reaction mechanism. Food Sci. Technol. Res., 9, 128-133 (2003).

8) Sawai Y. NMR analytical approach to clarify the molecular mechanisms of the antioxidative and radical-scavenging activities of antioxidants in tea: reaction of polyphenols with stable free radicals. Bulletin of the National Institute of Vegetable and Tea Science, 6, 23-58 (2007).

9) Okuda J, Miwa I, Inagaki K, Horie T, Nakayama M. Inhibition of aldose reductases from rat and bovine lenses by flavonoids. Biochem. Pharmacol., 31, 3807-3822 (1982).

10) Kanaya S, Goto K, Hara Yo, Hara Yu. The physiological effects of tea catechins on human volunteers. Proc. Intern. Sympo. Tea Science, 1991, 314-317 (1991).

11) Hara $Y$, Honda M. The inhibition of $\alpha$-amylase by tea polyphenols. Agric. Biol. Chem., 54, 1939-1945 (1990).

12) Honda $M$, Hara $Y$. Inhibition of rat small intestinal sucrase and $\alpha$-glucosidase activities by tea polyphenols. Biosci. Biotechnol. Biochem., 57, 123-124 (1993).

13) Yoshikawa M, Matsuda H. Saponins in food. Feedstuffs and medicinal plants. (Oleszek W, Marston A ed.) Kluwen Academic Publishers, pp. 189-203 (2000).

14) Chao J, Huo TI, Cheng HY, Tsai JC, Liao JW, Lee MS, Qin XM, Hsieh MT, Pao LH, Peng WH. Gallic acid ameliorated impaired glucose and lipid homeostasis in high fat diet-induced NAFLD mice. PLOS ONE, 9, e96969 (2014).

15) Takemoto M. Prevention and amelioration agent of lifestyle-related disease. Japanese Patent 5614674, 2014.9.19. 